\title{
Solvation Dynamics of 4-Aminophthalimide in Water-in-Oil Microemulsion of Triton X-100 in Mixed Solvents
}

\author{
Debabrata Mandal, Anindya Datta, Samir Kumar Pal, and Kankan Bhattacharyya* \\ Department of Physical Chemistry, Indian Association for the Cultivation of Science, Jadavpur, \\ Calcutta 700 032, India
}

Received: January 24, 1998

\begin{abstract}
Solvation dynamics of 4-aminophthalimide (4-AP) in water-in-oil microemulsion of Triton X-100 (TX) in mixed solvents containing 30\% benzene and $70 \% n$-hexane is studied using picosecond time-resolved Stokes shift. The steady-state emission spectrum of 4-AP in this microemulsion is very different from that in TX reverse micelle or in water. This indicates that in this microemulsion the 4-AP molecule stays in the water pool and the micropolarity of the water pool is very much different from that of ordinary bulk water. In TX microemulsions 4-AP exhibits wavelength-dependent fluorescence decays and a growth in the nanosecond time scale at long wavelengths. The solvation dynamics of 4-AP in TX microemulsion is bimodal with a short component of $0.74 \mathrm{~ns}$ and a very long component of $29.73 \mathrm{~ns}$. This is similar to the solvation dynamics of coumarin 480 in the large water pools of sodium dioctyl sulfosuccinate (AOT)/n-heptane/water microemulsions.
\end{abstract}

\section{Introduction}

Water molecules confined in nanodimensional environments play a fundamental role in many natural and biological processes. Ordinary water molecules exhibit solvation dynamics in the subpicosecond time scale. ${ }^{1}$ However, in many organized media such as cyclodextrins, ${ }^{1 \mathrm{a}, \mathrm{b}}$ reverse micelles, ${ }^{2 \mathrm{a}-\mathrm{c}}$ or micelles, ${ }^{2 \mathrm{~d}}$ the solvation dynamics of water molecules occurs in a time scale slower by several orders of magnitude. Recent dielectric relaxation and pulsed NMR studies of the biological systems reveal that while the dielectric relaxation time of ordinary water is about $10 \mathrm{ps}$, in many biological systems the dielectric relaxation of water is bimodal with a fast component of about $10 \mathrm{ps}$ and a 1000 times slower component of about $10 \mathrm{~ns} .^{3}$ The solvation time differs from the dielectric relaxation time by a factor of $\epsilon_{\infty} / \epsilon_{0}$, where $\epsilon_{\infty}$ and $\epsilon_{0}$ are respectively the dielectric constants at high and zero (static) frequencies. ${ }^{4}$ The 1000 times slower dielectric relaxation time and the 2-3 times lower static polarity $\left(\epsilon_{0}\right)$ of the organized assemblies compared to water cause the dramatic reduction in the solvation dynamics. The bimodal dielectric relaxation behavior of water in the organized assemblies was initially attributed to the presence of two kinds of water molecules, "bound" and "free", relaxing respectively in the nano- and picosecond time scales. ${ }^{3}$ However, very recently Nandi and Bagchi proposed a model according to which the slow component of the dielectric relaxation arises not from the inherent slowness of the bound water molecules but from the equilibration between the bound and free water. ${ }^{1 c}$ Surprisingly, in the case of semirigid gel (orthosilicate ${ }^{5 a}$ and polyacrylamide ${ }^{5 b}$ ) with very high bulk viscosity, it is observed that the solvation dynamics is very fast and occurs in the time scale of less than $50 \mathrm{ps}^{5}$

The water-in-oil microemulsions have recently been demonstrated to be an excellent system to study the relaxation behavior of water molecules in confined environments. ${ }^{2 a-c}$ In nonpolar media many surfactants aggregate with their polar group

* Corresponding author. E-mail: pckb@mahendra.iacs.res.in. Fax: 9133-473-2805. pointing inward to form a reverse micelle. On addition of water initially the polar headgroups of the surfactants get hydrated. On further addition of water the water pool is formed. Such a system containing nanodimensional water droplets ("water pool") enclosed by a layer of surfactant molecules and dispersed in a nonpolar hydrocarbon is known as a microemulsion. ${ }^{6-8}$ Earlier works on solvation dynamics in microemulsions are restricted to an ionic surfactant, sodium dioctyl sulfosuccinate (AOT). ${ }^{2}$ In the AOT microemulsion the abundant water molecules appear to be the best candidates for solvating an instantaneously created dipole. However, in view of the recent reports on nanosecond ionic solvation, ${ }^{9}$ one cannot rule out solvation by the sodium ions present in the water pool of a microemulsion. To eliminate the role of the ions, it is necessary to study solvation dynamics in a microemulsion comprising of neutral surfactants. Fortunately, several microemulsions based on neutral surfactants have been reported, recently. ${ }^{8}$ Of these, the microemulsions involving Triton X-100 (TX) in a mixture of $30 \%$ benzene and $70 \% n$-hexane have been reported to solubilize very high amounts of water. ${ }^{8 \mathrm{a}, \mathrm{b}}$ The hydrodynamic diameter of $0.27 \mathrm{M}$ TX in benzene/ $n$-hexane mixture $(3: 7, \mathrm{v} / \mathrm{v})$ increases from $7.2 \mathrm{~nm}$ in the absence of water to about $18 \mathrm{~nm}$ in the presence of water at a water to TX ratio, $R$, equal to 5 . For $R>8$, the hydrodynamic diameter increases steeply, and above 9 the solution becomes turbid due to the phase separation. ${ }^{8 \mathrm{a}, \mathrm{b}}$ The TX microemulsion has been studied so far by dynamic light scattering, turbidity measurements, and absorption spectroscopy of solvatochromic dyes. ${ }^{8}$ In the present work, we report on the solvation dynamics of 4-aminophthalimide (4-AP) in TX microemulsions in benzene/ $n$-hexane mixture.

\section{Experimental Section}

4-AP (Kodak) was purified by repeated recrystallization from a 1:1 alcohol-water mixture. All solvents were of spectroscopy grade and were distilled before experiment. Triton X-100 (TX, Aldrich) was used as received. The TX microemulsions in $30 \%$ 


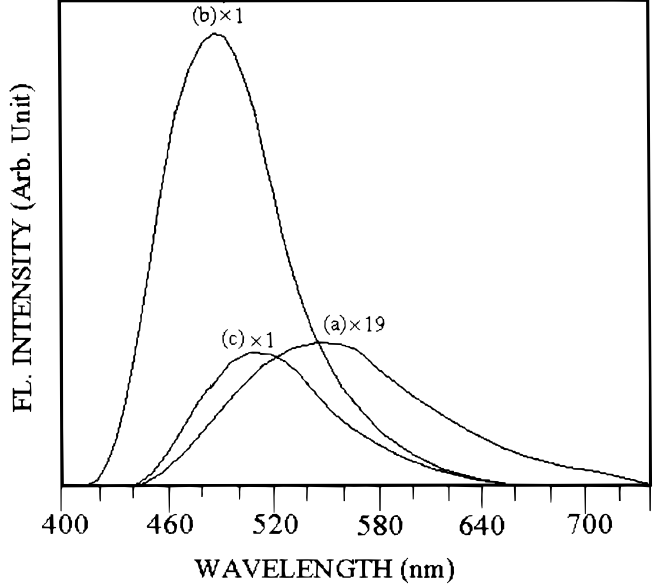

Figure 1. Emission spectra of 4-AP in (a) water, (b) $0.27 \mathrm{M}$ TX in benzene/ $n$-hexane (3:7, v/v), $R=0$, and (c) $0.27 \mathrm{M}$ TX in benzene/ $n$-hexane $(3: 7, \mathrm{v} / \mathrm{v}), R=5$.

benzene and $70 \% n$-hexane were prepared using literature procedure. ${ }^{2,8}$ Briefly, solid 4-AP was added to a $0.27 \mathrm{M}$ TX solution in benzene/ $n$-hexane mixture, and then the requisite amount of water was added by a microliter syringe. The laser system and the single-photon counting apparatus are described in earlier publications. ${ }^{2 b-d}$ The response time of the setup was about 60 ps. The fluorescence decays were deconvoluted using the global lifetime analysis software (PTI). Since the decays are biexponential with one component $<1 \mathrm{~ns}$ and another of $\sim 10 \mathrm{~ns}$, the decays were recorded at different full scales (48 and $6 \mathrm{~ns})$. The wavelength of excitation for the time-resolved studies was $300 \mathrm{~nm}$.

\section{Results}

Steady-State Emission. In aqueous solution, emission of 4-AP is very weak with emission maximum $\lambda_{\mathrm{em}}{ }^{\max }=550 \mathrm{~nm}$ and quantum yield $\phi_{\mathrm{f}}=0.01$, while in a nonpolar medium (e.g., dioxane) 4-AP shows intense emission with $\lambda_{\mathrm{em}}{ }^{\max }=435 \mathrm{~nm}$ and $\phi_{\mathrm{f}}=0.7 .^{2 \mathrm{c}, 10} 4$-AP is very sparingly soluble in benzene/ $n$-hexane mixture $(3: 7, \mathrm{v} / \mathrm{v})$, and the trace amount of 4 -AP present exhibits an emission maximum at around $425 \mathrm{~nm}$. In $0.27 \mathrm{M}$ TX in $30 \%$ benzene $/ 70 \% n$-hexane mixture, 4 -AP displays strong emission with $\lambda_{\mathrm{em}}{ }^{\max }=490 \mathrm{~nm}, \phi_{\mathrm{f}}=0.42$, and negligible intensities in the $410-430 \mathrm{~nm}$ region (Figure 1). This indicates that in the presence of TX, almost all the 4-AP molecules reside in the relatively polar region within the reverse micelle. On addition of water to the TX reverse micelles at a water-to-surfactant ratio $R=5$, the emission maximum of 4-AP shifts to $510 \mathrm{~nm}$ and the quantum yield decreases nearly 3 times to 0.13 (Figure 1). However, even at $R=5, \lambda_{\mathrm{em}}{ }^{\max }$ of 4 - $\mathrm{AP}$ is blue-shifted by $40 \mathrm{~nm}$ and $\phi_{\mathrm{f}}$ is 13 times higher compared to those in ordinary water. This indicates that the water pool of the TX microemulsions is substantially less polar than ordinary water. This is consistent with the earlier micropolarity measurements of this microemulsion using a solvatochromic betaine dye. ${ }^{8 a}$ It may be noted that position of the emission maximum of 4-AP in TX microemulsions is close to that in tert-butyl alcohol. ${ }^{10}$

Time-Resolved Emission. In the TX reverse micelles and microemulsions, the fluorescence decays of 4-AP are found to be wavelength-dependent. At the blue end ( $440-490 \mathrm{~nm})$, only a fast decay is observed, while at the red end $(500-650 \mathrm{~nm})$, the decays are much longer and are preceded by a growth in the nanosecond time scale (Figure 2). Such a wavelength dependence of the fluorescence decays indicates that 4-AP
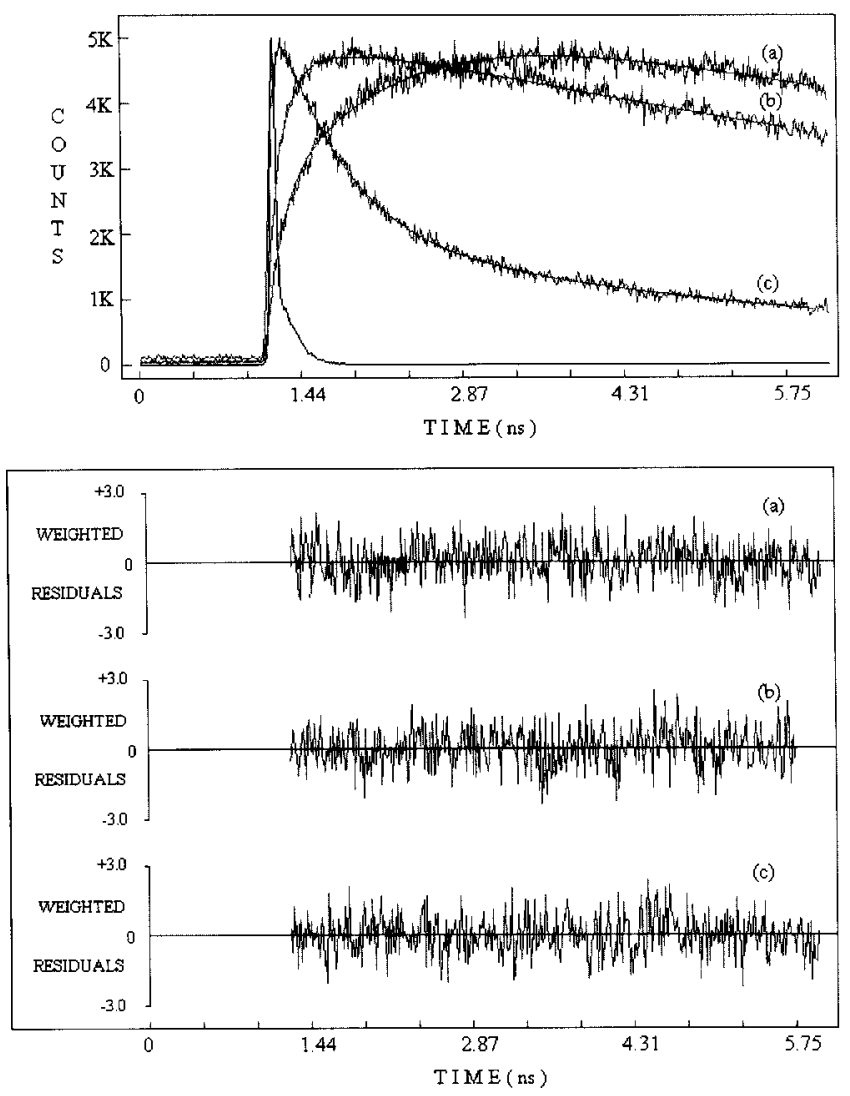

Figure 2. Fluorescence decays of 4-AP in $0.27 \mathrm{M}$ TX in benzene $/ n$ hexane $(3: 7, \mathrm{v} / \mathrm{v}), R=5$, at (a) $600 \mathrm{~nm}$, (b) $510 \mathrm{~nm}$, and (c) $450 \mathrm{~nm}$.

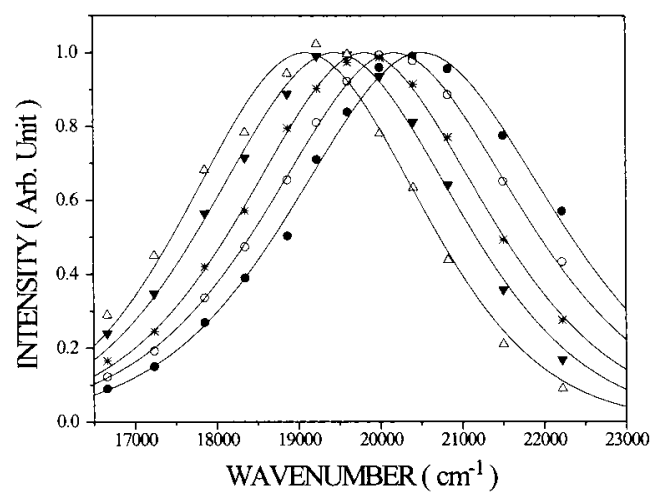

Figure 3. Time-resolved emission spectra of 4-AP in $0.27 \mathrm{M}$ TX in benzene $/ n$-hexane (3:7, v/v), $R=5$, at (a) $0 \mathrm{ps}(\bullet)$, (b) $325 \mathrm{ps}(\mathrm{O})$, (c) $1000 \mathrm{ps}(*)$, (d) $10000 \mathrm{ps}(\boldsymbol{\nabla})$ and (e) $50000 \mathrm{ps}(\triangle)$.

exhibits time-dependent Stokes shift in the TX reverse micelles and microemulsions. In the TX reverse micelles, in the absence of water, the lifetime of 4-AP is very long $(\sim 20 \mathrm{~ns})$ and the maxima of the time-resolved emission spectra exhibit very poor convergence. Thus the present discussion is restricted to TX microemulsions with $R=5$, which corresponds to reasonably large water pools. From the parameters corresponding to the best fits to the fluorescence decays, time-resolved emission spectra (Figure 3) are constructed and the emission maxima at different times are extracted by fitting to a log-normal function following the procedure of Fleming and Maroncelli. ${ }^{4 a}$ Using the emission frequencies $v(0), v(t)$, and $v(\infty)$ at time $0, t$, and $\infty$, respectively, the solvent response function $C(t)$, defined by

$$
C(t)=\frac{v(t)-v(\infty)}{v(0)-v(\infty)}
$$




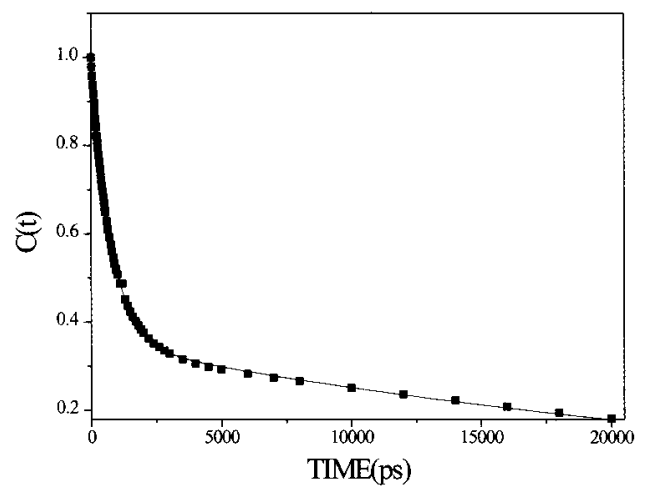

Figure 4. Decay of the response function, $C(t)$, of $4-\mathrm{AP}$ in $0.27 \mathrm{M}$ TX in benzene $/ n$-hexane $(3: 7, \mathrm{v} / \mathrm{v}), R=5$. The points denote the actual values of $C(t)$, and the solid line denotes the best fit to a biexponential decay.

TABLE 1: Decay Characteristics of the Solvent Response Function, $C(t)$, of 4-AP in 0.27 M TX Microemulsions in Benzene $(30 \%) / n$-Hexane $(70 \%)$ Mixture at $R=5$

\begin{tabular}{ccccc}
\hline$\Delta v\left(\mathrm{~cm}^{-1}\right)$ & $a_{1}$ & $\tau_{1}(\mathrm{~ns})$ & $a_{2}$ & $\tau_{2}(\mathrm{~ns})$ \\
\hline 1427 & 0.65 & 0.74 & 0.35 & 29.73
\end{tabular}

is constructed. Finally, the solvation times are obtained from the decay of $C(t)$ with time (Figure 4$)$. The decay characteristics of $C(t)$ are summarized in Table 1. It is readily seen that the solvation dynamics of 4-AP in the TX microemulsion is biexponential with one component of $0.74 \mathrm{~ns}$ and another of $29.73 \mathrm{~ns}$.

\section{Discussion}

The steady-state emission spectra of 4-AP in TX microemulsion $\left(\lambda_{\mathrm{em}}{ }^{\max }=490 \mathrm{~nm}\right)$ is very much different from that in benzene $/ n$-hexane mixture $\left(\lambda_{\mathrm{em}}{ }^{\max }=425 \mathrm{~nm}\right)$. Thus it is evident that in the TX microemulsion 4-AP experiences a highly polar microenvironment which is very much different from the hydrocarbon (benzene/ $n$-hexane) region of the microemulsion and that the probe 4-AP molecules are located in the inner water pool of the microemulsion. Though aromatic hydrocarbons are known to cause solvation dynamics, ${ }^{11 a, b}$ in this case, solvation by benzene can be ruled out because the probe 4-AP molecule resides inside the water pool away from benzene. Since in the polar water pool of TX microemulsions no ions are present, the role of ionic solvation in the nanosecond solvation dynamics observed in the microemulsions, also, appears to be minor. We have studied solvation dynamics of 4-AP in the TX microemulsions in benzene $/ n$-hexane mixture in the presence of $0.4 \mathrm{M}$ $\mathrm{CaCl}_{2}$ and found that the dynamics is almost identical to that in the absence of the salt. This once again demonstrates the very minor role of ionic solvation in the overall solvation dynamics in the microemulsions. The observed nanosecond solvation dynamics seems to be due to the water molecules and the polar headgroups of the surfactant (TX) molecules. It may be recalled that the observed bimodal solvation dynamics is similar to that observed for coumarin 480 in large water pools of the AOT microemulsions. ${ }^{2 c}$ For AOT microemulsions containing large water pool of radius $\sim 64 \AA$, the solvation dynamics of coumarin 480 exhibits two components, one of $\sim 2 \mathrm{~ns}$ and another of $\sim 12 \mathrm{~ns}^{2 \mathrm{c}}$ In the present case of 4-AP, in the TX microemulsion, the two components are respectively 0.74 and 29.73 ns. It is readily seen that both the components are very much slower than the relaxation time of ordinary water. The fast component $(0.74 \mathrm{~ns})$ is actually quite close to the solvation dynamics observed in Triton X-100 micelles in water
(0.72 ns for $4-\mathrm{AP}^{2 \mathrm{e}}$ and $1.46 \mathrm{~ns}$ for coumarin $\left.480^{2 \mathrm{~d}}\right)$. Thus the fast component can be attributed to the water molecules in the water pool. The very slow component of about $30 \mathrm{~ns}$ is rather intriguing. Following Nandi and Bagchi, ${ }^{1 \mathrm{c}}$ one may be tempted to ascribe the bimodal decay with 0.74 and 29.73 ns components to the equilibrium between the free and bound water. ${ }^{10}$ However, in the present case, one cannot rule out totally the effect of the polar headgroups of the surfactant molecules. The chain dynamics of the polymers occurs in the time scale of 100 ns. ${ }^{11 \mathrm{c}}$ If, accordingly, one assumes a dielectric relaxation time of $100 \mathrm{~ns}$ for the surfactants, a 30-ns component of solvation dynamics may very well result because, as discussed earlier, for such systems, $\epsilon_{\infty} / \epsilon_{0} \approx 4-6$. $^{2 \mathrm{c}}$

\section{Conclusions}

The present work shows that 4-AP is a sensitive fluorescent probe for the microenvironment of the TX microemulsions. From the steady-state and time-resolved studies, it is inferred that the probe 4-AP molecule is located in the water pool inside the microemulsions. The wavelength-dependent emission decays and the time-resolved Stokes shift indicate that the solvation dynamics of 4-AP in the water pool of TX microemulsion is bimodal with two components of 0.74 and 29.73 ns. The fast component is attributed to the water molecules present in the water pool. The very long component may be due either to an equilibrium between the free and bound water in the confined water pool or to the dynamics of the polar headgroups of the surfactant molecules. Since the observed dynamics of 4-AP in TX microemulsions is very similar to that in the microemulsion containing ionic surfactants, ${ }^{2 a-c}$ it appears that the role of ionic solvation in the solvation dynamics in the confined water pool is rather minor.

Acknowledgment. Thanks are due to the Department of Science and Technology, Government of India, and the Council of Scientific and Industrial Research (CSIR) for generous research grants. CSIR is also thanked for providing fellowships to A.D., D.M., and S.K.P. K.B. thanks Prof. Biman Bagchi for many stimulating discussions and for sending important preprints prior to publication.

\section{References and Notes}

(1) (a) Vajda, S.; Jimenez, R.; Rosenthal, S.; Fidler, V.; Fleming, G. R.; Castner, E. W., Jr. J. Chem. Soc., Faraday Trans. 1995, 91, 867. (b) Nandi, N.; Bagchi, B. J. Phys. Chem. 1996, 100, 13914. (c) Nandi, N.; Bagchi, B. J. Phys. Chem. B 1997, 101, 10954. (d) Zhu, S. B.; Singh, S.; Robinson, G. W. Adv. Chem. Phys. 1994, 85, 627. (e) Schwartz, B. J.; Rossky, P. J. J. Chem. Phys. 1996, 105, 6997. (f) Agmon, N. J. Phys. Chem. 1990, 100, 1073. (g) Nandi, N.; Roy, S.; Bagchi, B. J. Chem. Phys. 1995, 102, 1390. (h) Bagchi, B.; Chandra, A. Adv. Chem. Phys. 1990, 80, 1.

(2) (a) Lundgren, J. S.; Heitz, M. P.; Bright, F. V. Anal. Chem., 1995, 67, 3775. (b) Sarkar, N.; Das, K.; Datta, A.; Das, S.; Bhattacharyya, K. J. Phys. Chem. 1996, 100, 10523. (c) Das, S.; Datta, A.; Bhattacharyya, K. J. Phys. Chem. A 1997, 101, 3299. (d) Sarkar, N.; Datta, A.; Das, S.; Bhattacharyya, K. J. Phys. Chem. 1996, 100, 15483. (e) Datta, A.; Mandal, D.; Pal, S. K.; Das S.; Bhattacharyya, K. J. Mol. Liq. 1998, 77, 121.

(3) (a) Belton, P. S. J. Phys. Chem. 1995, 99, 17061. (b) Mashimo, S.; Kuwabara, S.; Yagihara, S.; Higashi, K. J. Phys. Chem. 1987, 91, 6337. (c) Menzel, K.; Rupprecht, A.; Kaatze, U. J. Phys. Chem. B 1997, 101 1255. (d) Urry, D. W.; Peng, S.; Xu, J.; McPherson, D. T. J. Am. Chem Soc. 1997, 119, 1161. (e) Pethig, R. in Protein-Solvent Interactions; Gregory, R. D., Ed.; Marcel-Dekker: New York, 1995; p 265.

(4) (a) Maroncelli, M.; Fleming, G. R. J. Chem. Phys. 1987, 86, 6224. (b) Jimenez, R.; Fleming, G. R.; Kumar, P. V.; Maroncelli, M. Nature 1994, 369, 471. (c) Maroncelli, M. J. Mol. Liq. 1993, 57, 1. (d) Reid, P. J.; Silva, C.; Barbara, P. F. J. Phys. Chem. 1995, 99, 3554. (e) Rossky, P. J.; Simon, J. D. Nature 1994, 370, 263.

(5) (a) Jordan, J. D.; Dunbar, R. A.; Bright, F. V. Anal. Chem. 1995, 67, 2436. (b) Datta, A.; Das, S.; Mandal, D.; Pal, S. K.; Bhattacharyya, K. Langmuir 1997, 13, 6922. 
(6) (a) Luisi, P. L. Angew. Chem., Int. Ed. Engl. 1985, 24, 439. (b) Fendler, J. H. Annu. Rev. Phys. Chem. 1984, 35, 137. (c) Moulik, S. P. Mukherjee, K. Proc. Indian Natl. Sci. Acad. 1996, 62A, 215. (d) Jean, Y. C.; Ache, J. H. J. Am. Chem. Soc. 1978, 100, 984, 6320. (e) Kotlarchzyk, M.; Huang, J. S.; Chen, S.-H. J. Phys. Chem. 1985, 89, 4382. (f) Cho, C. B.; Chung, M.; Lee, J.; Nguyen, T.; Singh, S.; Vedamuthu, M.; Yao, S.; Zhu, J.-B.; Robinson, G. W. J. Phys. Chem. 1995, 99, 7806.

(7) (a) Cassin, G.; Badiali, J. P.; Pileni, M. P. J. Phys. Chem. 1995 , 99, 12941. (b) Borsarelli, C. D.; Braslavsky, S. E. J. Phys. Chem. B 1997, 101, 6036. (c) Eastoe, J.; Young, W. K.; Robinson, B. H. J. Chem. Soc. Faraday Trans. 1990, 86, 2883. (d) Jain, T. K.; Varshney, M.; Maitra, A J. Phys. Chem. 1989, 93, 7409. (e) Mukherjee, K.; Mukherjee, D. C.; Moulik, S. P. Langmuir 1993, 9, 1727. (f) Gehlan, M. H.; De Schryver, F. C.; Dutt, G. B.; Van Stan, J.; Boens, N.; Auweraer, M. J. Phys. Chem. 1995, 99, 14407.
(8) (a) Zhu, D.-M.; Wu, X.; Schelly, Z. A. J. Phys. Chem. 1992, 96, 7121. (b) Zhu, D.-M.; Wu, X.; Schelly, Z. A. Langmuir 1992, 8, 1538. (c) Zhu, D.-M.; Wu, X.; Schelly, Z. A. Langmuir 1992, 8, 48. (d) Zhu, D.-M.; Wu, X.; Schelly, Z. A. J. Phys. Chem. 1992, 96, 2382. (e) Caldaru, H.; Caragheorgheopal, A.; Dimonie, M.; Donescu, D.; Dragutan, I.; Marinescu, N. J. Phys. Chem. 1992, 96, 7109.

(9) (a) Bart, E.; Melstein, A.; Huppert, D. J. Phys. Chem. 1995, 99 9253. (b) Neria, E.; Nitzan, A. J. Chem. Phys. 1994, 100, 3855. (c) Chandra, A.; Wei, D.; Pattey, G. N. J. Chem. Phys. 1993, 98, 4959.

(10) (a) Soujanya, T.; Fessenden, R. W.; Samanta, A. J. Phys. Chem. 1996, 100, 3507. (b) Pryor, B. A.; Palmer, P. M.; Andrews, P. M.; Berger, M. B.; Troxler, T.; Topp, M. R. Chem. Phys. Lett. 1997, 271, 19.

(11) (a) Bagchi, B. J. Chem. Phys. 1994, 100, 6658. (b) Fourkas, J. T.; Benigno, A.; Berg, M. J. Chem. Phys. 1993, 99, 8552. (c) Cassol, R.; Ge, M.-T.; Ferrarini, A.; Freed, J. H. J. Phys. Chem. B 1997, 101, 8782. 